Research Paper

\title{
Modulation of Wnt Activity and Cell Physiology by Butyrate in LT97 Microadenoma Cells
}

\author{
Darina Lazarova1, Andrew Lee1, Terrence Wong1, Brigitte Marian², Christopher Chiaro'1, Christian Rainey³, \\ Michael Bordonaro ${ }^{1 凶}$ \\ 1. Department of Basic Sciences, the Commonwealth Medical College, 525 Pine Street, Scranton, PA 18509, USA. \\ 2. Medizinische Universität Wien, Institut für Krebsforschung, Borschkegasse 8a, 1090 Wien, Austria. \\ 3. Marywood University, 2300 Adams Avenue, Scranton, PA 18509, USA. \\ $\triangle$ Corresponding author: The Commonwealth Medical College, 525 Pine Street, Scranton, PA 18509. Tel: 570-504-9646 Fax: 570-504-9636 \\ Email: mbordonaro@tcmedc.org.
}

() Ivyspring International Publisher. This is an open-access article distributed under the terms of the Creative Commons License (http://creativecommons.org/ licenses/by-nc-nd/3.0/). Reproduction is permitted for personal, noncommercial use, provided that the article is in whole, unmodified, and properly cited.

Received: 2014.0I.I3; Accepted: 2014.01.25; Published: 20I4.02.14

\begin{abstract}
Dietary fiber intake is linked to a reduced risk of colon cancer. This effect may in part be due to butyrate, the fermentation product of fiber in the colon. Butyrate is a short-chain fatty acid that acts as a histone deacetylase inhibitor (HDACi). Butyrate induces apoptosis and represses clonal growth of colorectal cancer (CRC) cells, in a manner dependent upon the hyperactivation of Wnt /beta-catenin signaling. While fiber has been linked to CRC prevention, in vitro studies on the action of butyrate have used CRC cell lines, instead of cells representative of earlier stages of colonic neoplasia, which are the likely target of butyrate-mediated preventive activity. The LT97 cell line is derived from a microadenoma, the earliest stage of colonic neoplasia from which cells can be isolated. We characterized LT97 cells with respect to effects of butyrate on Wnt signaling and apoptosis, and we determined whether modulation of CREB binding protein (CBP)/p300 activity influences the ability of butyrate to induce Wnt activity and apoptosis. We report that in LT97 cells, butyrate induces apoptosis, strongly upregulates Wnt signaling, and the upregulation of Wnt signaling is dependent upon CBP/p300 activity. In addition, findings from overexpression experiments suggest differences between CBP and p300 in their ability to influence Wnt signaling in LT97 cells; $\mathrm{p} 300$, but not CBP, stimulates basal Wnt activity. We also evaluated differences in gene expression between early stage LT97 cells and late stage metastatic SW620 CRC cells that exhibit markedly different cellular phenotypes. The comparative gene expression analyses revealed differences that may impact neoplastic progression and the sensitivity to the effects of butyrate. The findings have implications for the prevention of CRC by fiber/butyrate.
\end{abstract}

Key words: LT97, microadenoma, colorectal cancer, butyrate, CBP, p300.

\section{Introduction}

Epidemiological studies have demonstrated an association between a high intake of dietary fiber and a reduced risk of colon cancer $(1,2)$. Butyrate, the breakdown product of soluble fiber in the colonic lumen, acts as a histone deacetylase inhibitor (HDACi) and may contribute to the protective effects of fiber (3-7). Butyrate stimulates apoptosis and inhibits clonal growth of colorectal cancer (CRC) cells in culture (7), and we have provided evidence that these effects of butyrate are in part explained by the hy- peractivation of canonical Wnt transcriptional activity by this agent (4-6).

Canonical Wnt activity results from the accumulation of active beta-catenin, which associates with DNA-binding Tcf factors to stimulate transcriptional activity from Wnt target genes (8-11). Constitutive activation of Wnt signaling, due to mutations in the $A P C$ and beta-catenin genes (8-11), promotes colonic cell proliferation and tumorigenesis. However, abnormally high levels of canonical Wnt signaling 
promote apoptosis (12). Butyrate hyperactivates Wnt signaling in CRC cells (4-6), and this activity of butyrate determines the levels of cellular apoptosis. The growth suppressive and apoptotic effects of butyrate linearly correlate with the upregulation of Wnt activity induced by this agent in ten human CRC cell lines (5). We have confirmed that the association between enhanced Wnt activity and both apoptosis and repressed clonal growth in butyrate-treated CRC cells is causative (4-6).

Butyrate is likely most effective against early stage colonic neoplasms (7); thus, intake of dietary fiber, a source of colonic butyrate, is linked to CRC prevention, and therefore, it must affect the early stages of the disease. However, in vitro studies on the action of butyrate have typically used fully transformed CRC cells that are not representative of the colonic cells targeted by butyrate in vivo. Therefore, it would be useful to evaluate the effects of butyrate on adenoma, rather than carcinoma, cells. Adenoma cell lines are typically difficult to establish and maintain in culture; however, the LT97 cell line has been successfully isolated from a microadenoma, the earliest colonic neoplasm from which cells can be cultured (13). This APC mutant cell line was isolated from a patient with hereditary familial adenomatous polyposis $(F A P)$, and is characterized by the presence of C-terminus truncated APC protein and a lack of full length, wild-type APC protein $(13,14)$. Consistent with the idea that butyrate is most effective against early stage colonic neoplasia, LT97 cells are more sensitive to the growth suppressive effects of butyrate compared to the CRC cell line HT-29 (14). Microarray data comparing LT97 and HT29 cells in the presence or absence of butyrate showed upregulation of a number of genes by butyrate, particularly those involved with detoxification pathways (e.g., glutathione S-transferases) (15).

The effects of butyrate on Wnt signaling in colonic cells may be influenced by various cotranscriptional factors that associate with beta-catenin-Tcf complexes. CREB binding protein (CBP) and p300 are histone acetylases (HATs) that associate with beta-catenin and influence Wnt signaling and apoptosis/growth of CRC cells (16-25). We have shown that modulation of CBP-mediated or p300-mediated Wnt signaling affects the ability of butyrate to induce Wnt activity and apoptosis in CRC cells, and does so in a cell-type specific manner $(26,27)$. These findings correlate with previous studies using the clinically relevant small molecule inhibitor ICG-001, which specifically blocks the association of CBP, but not p300, with beta-catenin (16). These studies demonstrated important differences between the action of CBP and p300 on Wnt signaling and cell physiology; thus,
CBP-mediated Wnt signaling is associated with cell proliferation; whereas, p300-mediated Wnt signaling is associated with cell differentiation (16-21).

The primary objective of the present study was to use LT97 cells to model the response of early stage colonic neoplasia to butyrate. In particular, our aims were to characterize the response of LT97 cells to butyrate in terms of (a) the effects of butyrate on Wnt activity and apoptosis, and (b) the role of $\mathrm{CBP} / \mathrm{p} 300$ activity in these effects. We also evaluated differences in gene expression between early stage adenoma LT97 cells and the late stage carcinoma SW620 cell line, which was derived from a lymph node CRC metastasis.

Here we demonstrate, for the first time, that LT97 cells exhibit a marked sensitivity to butyrate with respect to the modulation of Wnt signaling, and that Wnt signaling hyperactivation in early stage neoplastic cells is dependent upon CBP/p300-mediated activity. LT97 cells are also highly sensitive to the apoptosis-inducing activity of butyrate; however, the combination of butyrate and ICG-001 results in the highest levels of LT97 cell apoptosis. We have also identified marked changes in gene expression between LT97 adenoma cells and SW620 metastatic cells. These findings have important implications for prevention of CRC by fiber/butyrate.

\section{Materials and Methods}

\section{Cells}

SW620 cells were obtained from ATCC, and cultured as previously described $(4,5)$. LT97 cells were obtained from Dr. Bridgette Marian (Medical University of Vienna, Austria), and cultured essentially as described $(13,14)$.

\section{Plasmids, cell lines, transfection, luciferase assay}

pTOPFLASH (TOP) and pFOPFLASH (FOP) were obtained from Dr. H. Clevers (UMC Utrecht, Utrecht, Netherlands). KLF4 expression vector was obtained from Dr. Mark Feinberg (Brigham and Women's Hospital, Boston, MA). Expression vectors for p300 and CBP were obtained from Addgene. For LT97 cells, typically, 10,000 cells were plated per well in a 96 well plate and four days later the transfections were performed; transfection with lipofectamine 2000 and luciferase assays were performed as previously described (4-6).

\section{Reagents}

Butyrate was obtained from Sigma, ICG-001 was from the Kahn laboratory and Selleckchem; C646 was from EMD Millipore Chemicals. 


\section{Total Human Genome Microarray Analysis}

After treatment with or without $5 \mathrm{mM}$ butyrate for $17.5 \mathrm{hr}$, cells were washed with $1 \times$ PBS, scraped into PBS and pelleted; the pellets were snap frozen in liquid nitrogen and sent to Genus Biosystems for RNA extraction and microarray analyses, utilizing the Agilent human whole genome oligo microarray. RNA extraction and array analyses were performed by Genus as follows. RNA was extracted and purified with Ambion Ribopure isolation, with RNA quality assessed by an Agilent Bioanalyzer. Following first and second strand cDNA synthesis, cRNA target was prepared, fragmented to a uniform size, and hybridized to Agilent Human v2 GE $4 \times 44 \mathrm{~K}$ arrays. Slides were subsequently washed and scanned on an Agilent G2565 Microarray Analyzer and the resulting data were analyzed with Agilent Feature Extraction and GeneSpring GX v7.3.1 software.

\section{Apoptosis and Proliferation}

Apoptotic analyses were performed using the caspase $3 / 7$ glo kit of Promega, as previously described $(26,27)$. Assays of cell proliferation/viability were performed with the QuickCell Proliferation Kit (Biovision). For proliferation assays, cells were plated out at 10,000 cells/well in 96-well plates, treated the following day and assayed $72 \mathrm{hr}$ later. Data were corrected for the background.

\section{Western blotting}

Western blotting was performed essentially as previously described (6). Antibodies were from Santa Cruz Biotechnology. Band images were adjusted for brightness and contrast to enhance visualization.

\section{Statistics}

Students T-test was utilized, with statistical significance set at $\mathrm{P}<0.05$.

\section{Results}

\section{Butyrate markedly upregulates Wnt activity and apoptosis in LT97 cells}

We had previously measured the induction of Wnt activity by butyrate in ten human CRC cell lines, nine of which exhibit constitutive Wnt signaling; in these nine cell lines, fold-induction of Wnt activity by butyrate ranged from a low of 1.4 (HT-29 cells) to a high of 21.8 (HCT-116 cells) (5). However, early stage neoplastic cells are likely more representative of the in vivo targets of the preventive activity of fiber-derived butyrate (7). We therefore evaluated the effects of butyrate on Wnt signaling and apoptosis in LT97 microadenoma cells (13). These early stage colonic neoplastic cells were previously shown to be more sensitive to the growth suppressive effects of butyrate compared to HT-29 CRC cells (14); however, the effects of butyrate on Wnt signaling and apoptosis in this microadenoma cell line had not previously been determined.

LT97 cells exhibited a markedly greater induction of Wnt activity by butyrate compared to the ten CRC cell lines we have previously analyzed. Thus, $17.5 \mathrm{hr}$ exposure of LT97 cells to $5 \mathrm{mM}$ butyrate resulted in a 43-fold $(\mathrm{P}<0.02)$ induction of Wnt/beta-catenin transcriptional activity (Fig.1A). We have previously shown that the ability of butyrate to promote CRC cell apoptosis, and repress CRC growth, is casually associated with the degree of Wnt hyperactivation induced by the agent. Therefore, based upon the 43-fold upregulation of Wnt activity by butyrate in LT97 cells, we hypothesized that LT97 cells would exhibit proportionally high fold induction of apoptosis upon exposure to butyrate. Measurement of caspase 3/7 activation, a hallmark of apoptosis, in LT97 cells exposed to $5 \mathrm{mM}$ butyrate revealed a 5.8 -fold induction of enzyme activity $(\mathrm{P}<0.005)$ (Fig.1B). In comparison, HCT-116 CRC cells exhibited a 2.6-fold induction of caspase $3 / 7$ activity (26). Thus, LT97 cells undergo high levels of apoptosis in the presence of butyrate, and this sensitivity to the apoptotic effects of butyrate is consistent with (a) the hyperactivation of Wnt/beta-catenin activity in the cells (Fig.1A), and (b) the butyrate-mediated growth suppression (14).

\section{Downregulation of CBP-mediated Wnt signaling by ICG-00I in LT97 cells}

The association between beta-catenin and the transcriptional coactivators CBP and p300 influences Wnt signaling (16-21). The small molecule inhibitor ICG-001 binds to CBP but not to p300; treatment of CRC cells with ICG-001 interferes with beta-catenin-CBP binding while maintaining, or even promoting, the association between beta-catenin and p300 (16). ICG-001 results in repressed Wnt activity as measured by reporter assays, while elevating caspase activity and inhibiting CRC cell growth (16).

We have previously shown (26) that the upregulation of Wnt activity in butyrate-treated CRC cells is dependent upon CBP-mediated Wnt signaling and can be abrogated by ICG-001 (16). Given the marked upregulation of Wnt signaling and caspase 3/7 activity in butyrate-treated LT97 cells, we evaluated whether these cells depend upon CBP activity for the hyperactivation of Wnt signaling. In the absence of ICG-001, a marked enhancement $(\mathrm{P}<0.01)$ of Wnt/beta-catenin activity by butyrate was observed. However, ICG-001 inhibited butyrate-mediated Wnt hyperactivation: in the presence of $75 \mu \mathrm{M}$ ICG-001 (26), butyrate treatment of LT97 cells enhanced Wnt 
activity by only 3.5-fold ( $\mathrm{P}<0.05)$ (Fig.2A). Therefore, similar to CRC cells, the ability of butyrate to upregulate Wnt signaling in LT97 microadenoma cells is dependent upon CBP activity, and can be repressed by ICG-001. We then determined the effects of ICG-001 treatment on the ability of butyrate to upregulate caspase activity. An efficient upregulation of caspase activity occurred in butyrate-treated LT97 cells (5.4-fold, P < 0.001), and treatment with ICG-001 alone increased caspase $3 / 7$ activity by 3.0 -fold in this cell line $(\mathrm{P}<0.001)$ (Fig.2B). Cotreatment with both agents resulted in a 6.0-fold increase in caspase activity compared to mock-treated cells $(\mathrm{P}<0.001)$. However, there was no statistically significant difference in the increase in caspase $3 / 7$ activity between ICG-001/butyrate- and butyrate-treated cells.

CBP-mediated Wnt activity may influence cell proliferation, as well as apoptosis (18-21, 26,27). In CRC cells, the combined treatment with ICG-001 and butyrate had the most profound suppressive effect on cell growth $(26,27)$. In LT97 cells, we observed (Fig.2C) that butyrate has a marked effect in suppressing cell growth $(\mathrm{P}<0.001)$, confirming previous reports (14). ICG-001 resulted in a moderate decrease in cell growth $(\mathrm{P}<0.03)$, and the combination of both agents led to the lowest levels of LT97 cell proliferation $(\mathrm{P}<0.001)$.

\section{A}

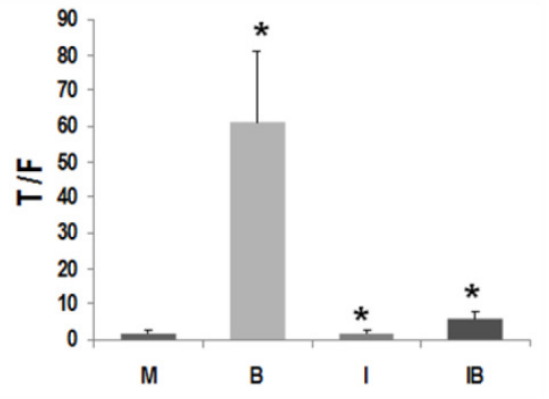

C

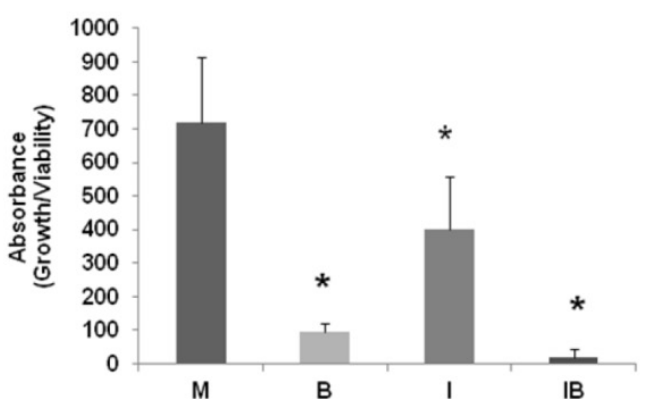

A

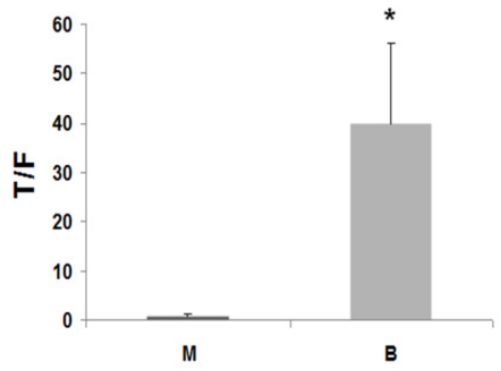

B

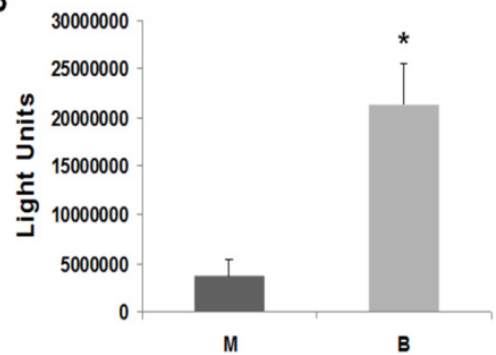

Figure I. Butyrate upregulates Wnt activity and apoptosis in LT97 microadenoma cells. (A) LT97 cells were transfected (lipofectamine 2000) with TOP/FOPFlash reporter vectors and with PRLTK for normalization of transfection efficiency. After 5 hours cells were mock treated (M) or treated with $5 \mathrm{mM}$ butyrate for $17.5 \mathrm{hr}(\mathrm{B})$. Wht signaling measured by the ratio of luciferase expression from TOPFlash $(T)$ to FOPFlash $(F)$ is shown. Data are from three separate experiments. (B) 10,000 LT97 cells/well were plated into a 96 well plate and allowed to grow for four days. Cells were then treated with $5 \mathrm{mM}$ butyrate (B) for $24 \mathrm{hr}$ or mock treated (M) and caspase activation was measured with the caspase 3/7 Glo luciferase kit (Promega). Background readings from medium alone are subtracted from the luciferase readings of the samples. Data are from three separate experiments. Bars, SDs. * = statistical significance.

B

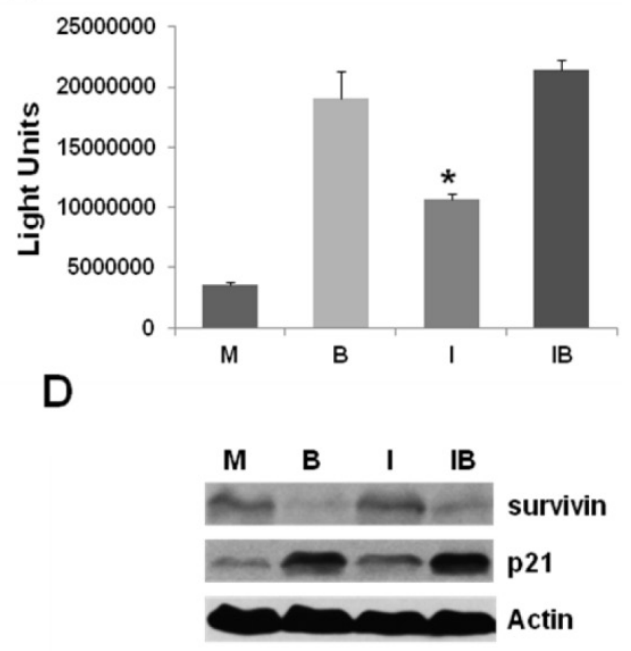

Figure 2. ICG-00I abrogates the upregulation of Wnt activity by butyrate in LT97 cells, but allows for butyrate-mediated apoptosis and repression of proliferation. (A) LT97 cells were transfected and treated as described in Fig IA, except that cells were also treated with 5 mM butyrate alone (B), $75 \mu$ M ICG-00I alone (I), both agents (IB), or mock treated (M). Data are from three separate experiments. (B) LT97 cells were treated as described in Fig IB, except that cells were treated with 5 mM butyrate alone (B), $75 \mu M$ ICG-00I alone (I), both agents (IB), or mock treated (M). Data are from three separate experiments. (C) LT97 cells have a doubling time of $\sim 72-96$ hr, in contrast to the more typical $\sim 24 \mathrm{hr}$ doubling time observed in most CRC cell lines $(13,14,15)$. Therefore, for butyrate/ICG-00I treatments, we assayed LT97 cell proliferation/viability $96 \mathrm{hr}$ after plating, which is $72 \mathrm{hr}$ after commencement of treatment with butyrate and/or ICG-00I. Thus, LT97 cells were treated with butyrate (B), ICG-00I (I), or both agents (IB) as described in Fig. 2B and cell viability/proliferation was assayed, after a further $72 \mathrm{hr}$, as previously described $(25,26)$. Data are from five separate experiments. Bars, SDs. $*=$ statistical significance. (D) Representative Western blot data showing expression of survivin and p2I in LT97 cells mock treated (M), treated with 5 mM butyrate (B), $75 \mu$ M ICG-00I (I), or both agents (IB) for $24 \mathrm{hr}$. Actin was used as a loading control. 
In SW620 CRC cells, expression of the anti-apoptotic factor survivin is suppressed by treatment with ICG-001 (26). Further, expression of the cell cycle inhibitor p21 is increased by butyrate, and this increase is abrogated by cotreatment with ICG-001 (26). Differences in the patterns of survivin and p21 expression likely mediate the cell-type specific effects of ICG-001/butyrate cotreatment on CRC cell proliferation and apoptosis (26). Thus, we ascertained how treatment with butyrate and/or ICG-001 modulates survivin and p21 expression in LT97 cells. Survivin expression in LT97 cells is decreased by butyrate treatment; however, unlike the observations in CRC cells (26), is unaffected by treatment with ICG-001 (Fig.2D). Expression of p21 is upregulated by butyrate in LT97 cells and, also unlike what has been observed in CRC cells (26), this increase of p21 expression is maintained in the presence of ICG-001 (Fig.2D).

\section{Modulation of CBP/p300-mediated Wnt signaling in LT97 cells}

To confirm the role of CBP/p300 activity in butyrate-mediated Wnt hyperactivation, we utilized a genetic modulator of this activity, Kruppel-like factor 4 (KLF4), as well as an additional pharmacological agent (C646). We also performed CBP/p300 overexpression experiments, similar to those performed in our previous analyses of CRC cells (27).

KLF4, which is expressed in the adult intestine and promotes intestinal differentiation, downregulates CBP/p300-mediated Wnt activity by inhibiting
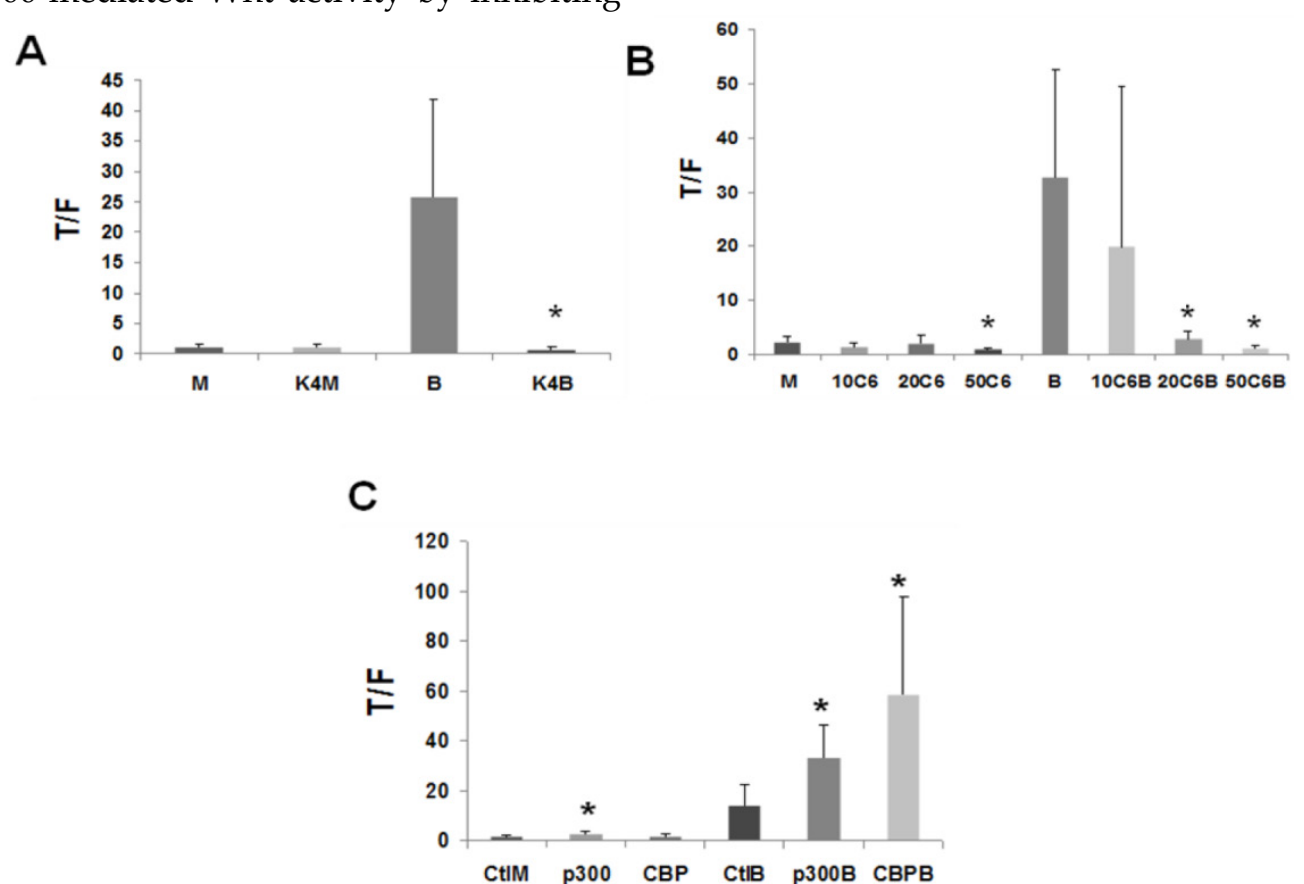

Figure 3. CBP/p300 activity influences Wnt signaling in LT97 cells. (A) LT97 cells were transfected as described in Fig. IA, except that KLF4 (K4) was cotransfected. Cells were treated with butyrate (B) or mock treated (M) and analyzed as described in Fig. I. Data are from seven separate experiments. (B) LT97 cells were transfected as in Fig. IA; after 5 hours cells were mock untreated (M) or treated ( $17.5 \mathrm{hr}$ ) with $5 \mathrm{mM}$ butyrate (B), or $10 \mathrm{mM}, 20 \mathrm{mM}$, or $50 \mathrm{mM}$ C646 (I0C6, $20 \mathrm{C} 6$, or $50 \mathrm{C} 6$, respectively), or combinations of butyrate with those concentrations of C646. Data are from four separate experiments. (C) LT97 cells were transfected as in Fig. IA and cotransfected with pcDNA3.I (Ctl) or P300 or CBP expression vectors. After 5 hours cells were left untreated (Ctl) or treated with $5 \mathrm{mM}$ butyrate for 17 hr (B), or mock treated (M). Data are from six separate experiments. Bars, SDs. $*=$ statistical significance. 
Downregulation of $\mathrm{CBP}$ or $\mathrm{CBP} / \mathrm{p} 300$ activity interferes with the ability of butyrate to induce Wnt signaling in LT97 cells (Figs.2, 3), and overexpression of p300, but not CBP, upregulated basal and butyrate-induced Wnt activity in CRC cells (27). Therefore, we investigated how CBP/p300 overexpression influences butyrate-mediated Wnt hyperactivation in the LT97 cell line. In LT97 cells, overexpression of both p300 and CBP influenced the ability of the HDACi butyrate to modulate Wnt signaling (Fig.3C). In the absence of butyrate treatment, overexpression of p300, but not CBP, resulted in a low upregulation of Wnt signaling $(\mathrm{P}<0.04)$. In the presence of $5 \mathrm{mM}$ butyrate, overexpression of $\mathrm{p} 300$ resulted in a more than two-fold enhancement of Wnt activity compared to butyrate treatment alone $(\mathrm{P}<0.02)$, while overexpression of CBP coupled with butyrate treatment resulted in a four-fold $(P<0.03)$ increase in Wnt activity compared to butyrate treatment alone.

\section{Gene expression analysis of adenoma LT97 and carcinoma SW620 cells}

Alteration of cell signaling pathways, leading to changes in gene expression, can significantly modify cellular phenotypes and influence neoplastic progression (32,33, and refs. therein). We therefore decided to compare changes in gene expression between the LT97 adenoma cell line and the SW620 CRC cell line.

In a previous survey of ten CRC cell lines, we observed that SW620 cells exhibit a moderate upregulation of Wnt activity and apoptosis by butyrate (5). There are three reasons for choosing SW620 cells, from this group of ten cell lines, to represent CRC cells in the current paper. First, a previous study (13) specifically compared SW620 cells to LT97 cells, demonstrating that the SW620 CRC cell line, but not the LT97 adenoma cell line, exhibits anchorage-independent growth. Second, SW620 metastatic CRC cells are commonly used for in vivo tumorigenesis experiments (e.g., mouse xenograft studies) and it is therefore particularly important to understand how butyrate influences gene expression in this cell line. Third, and most importantly, LT97 and SW620 cells represent the extremes of colonic neoplastic progression. LT97 cells, derived from a microadenoma, represent the earliest stage of neoplasia, while SW620 cells, derived from a lymph node metastasis of a colonic tumor, represent late stage disease (13 and refs. therein). We reasoned that a microarray comparison of these two cell lines would identify gene targets responsible for both the adenoma to carcinoma progression and the development of metastasis, as well as responsiveness to butyrate. The genes identified by this comparative study may therefore represent potential therapeutic targets.

We evaluated gene expression in these two cell lines via microarray analysis, to identify differences in basal and butyrate-modulated gene expression. A screening experiment consisting of a single replicate of a full human genome microarray analysis was performed to identify genes for subsequent validation by western blot analyses.

Figure 4 summarizes the differences in gene expression observed "within treatments" (comparing LT97 vs. SW620 cells for both mock and butyrate treatment, Fig.4A) and "within cell lines" (comparing mock- to butyrate-treated samples for each cell line, Fig.4B). For the "within treatment" analysis, 3512 genes exhibited a greater than three-fold difference in expression comparing mock-treated LT97 and SW620 cells, 2800 genes exhibited three-fold difference in butyrate-treated LT97 and SW620 cells, and 2784 genes exhibited three-fold difference with both mock and butyrate treatment (Fig.4A). There were also significant differences in gene expression observed in the "within cell line" analysis.

Thus, in LT97 cells, 3177 genes exhibited a greater than three-fold difference in expression comparing mock and butyrate treatment, and in SW620 cells, 3007 genes exhibited that pattern. In addition, there were a total of 2151 genes that exhibited a greater than three-fold difference in expression upon butyrate treatment in both cell lines tested (Fig.4B). Relative expression levels of a number of selected genes that influence or are influenced by Wnt signaling, and/or which may mediate neoplastic progression, are shown in Table 1 . We subsequently utilized western blot analyses to evaluate the expression of selected relevant genes at the protein level.

Table I. Differential expression of selected genes in untreated and butyrate treated LT97 and SW620 cells. Several gene targets are shown, with the fold-change in expression between cell lines in the absence or presence of $5 \mathrm{mM}$ butyrate. These data include changes in expression of both less than, and greater than, the three-fold change level required for inclusion in the Venn diagram (Fig. 5). A, absent (below level of detection).

\begin{tabular}{lllll}
\hline Gene & $\begin{array}{l}\text { Control } \\
\text { SW620/LT97 }\end{array}$ & $\begin{array}{l}\text { Butyrate } \\
\text { SW620/LT97 }\end{array}$ & $\begin{array}{l}\text { LT97 } \\
\text { Butyrate/ } \\
\text { Control }\end{array}$ & $\begin{array}{l}\text { SW620 } \\
\text { Butyrate/ } \\
\text { Control }\end{array}$ \\
\hline CCND2 & 0.07 & 1.13 & 0.86 & 14.52 \\
CDH1 & 0.06 & 0.27 & 1.39 & 6.42 \\
CREBBP & 0.13 & $\mathrm{~A}$ & 0.11 & $\mathrm{~A}$ \\
EP300 & 2.08 & 0.55 & 1.92 & 0.51 \\
KLF4 & 1.97 & 0.64 & 2.76 & 0.89 \\
MYC & 5.49 & 68.53 & 0.04 & 0.52 \\
SP5 & 155.42 & 120.39 & 2.6 & 1.97 \\
TLE1 & 0.27 & 0.15 & 1.67 & 0.57 \\
VIM & 2685.45 & 73.21 & 38.16 & 1.04 \\
\hline
\end{tabular}


Reporter data demonstrated the importance of $\mathrm{CBP} / \mathrm{p} 300-$ mediated Wnt activity, in colonic cells, including the LT97 line [Figs.2, 3. refs. $(25,26)]$. Our microarray data indicated that $\mathrm{CBP}$ is expressed at higher levels in mock-treated LT97 cells than in mock-treated SW620 cells, and this difference was confirmed by western blot analyses. The array findings also indicated that butyrate treatment of LT97 cells decreases CBP expression; however, this was not confirmed at the protein level (Fig.5A). Changes in
CBP expression after butyrate treatment in SW620 cells could not be quantitatively measured in the array analysis due to the very low $C B P$ mRNA expression; however, we consistently observed increased CBP expression at the protein level in butyrate-treated SW620 cells. Microarray data indicated minimal variation in p300 levels between the two cell lines, which was confirmed at the protein level, particularly in the presence of butyrate (Fig.5B).
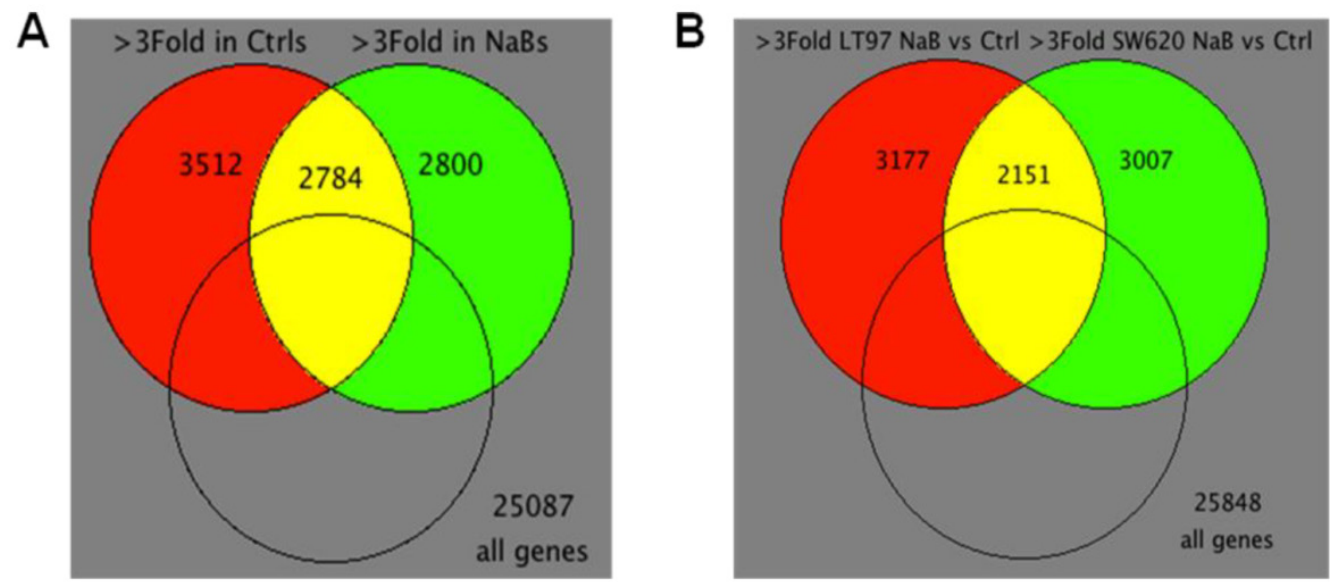

Figure 4. Venn diagrams of microarray data. (A) Venn diagram for "within treatment" comparison of differentially expressed genes in mock or butyrate treatments across cell lines (>3Fold, 9,096 probes). 99 intersection probes are differentially expressed in opposite directions. 2,685 intersection probes are differentially expressed in the same direction. (B) Venn diagram for "within cell lines" comparison of differentially expressed genes in mock treated control (Ctrl) vs. butyrate (NaB) treatments of two cell lines (>3Fold, 8,335 probes). 165 intersection probes are differentially expressed in opposite directions. I,986 intersection probes are differentially expressed in the same direction.

A

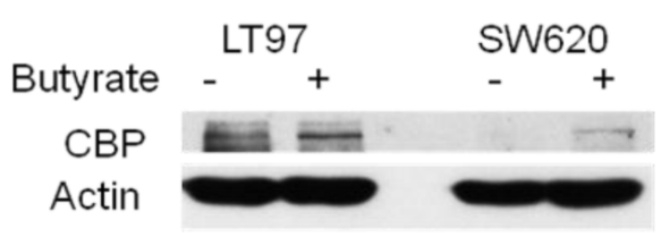

C

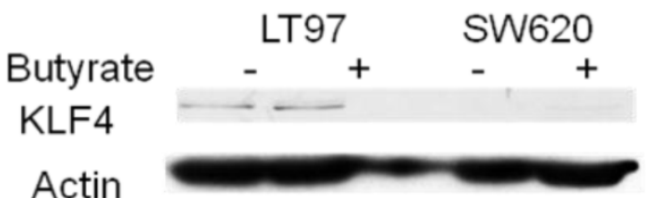

E

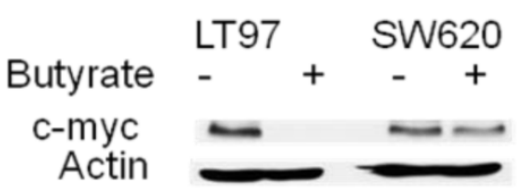

B

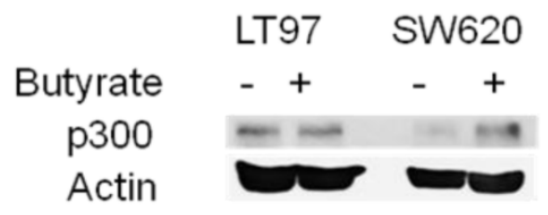

D

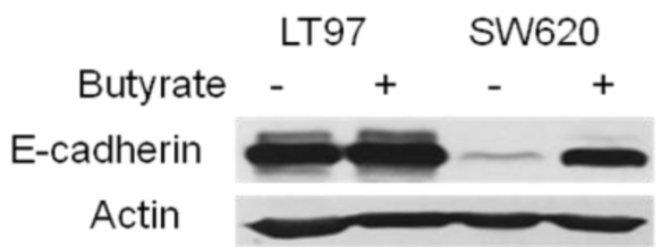

$\mathbf{F}$

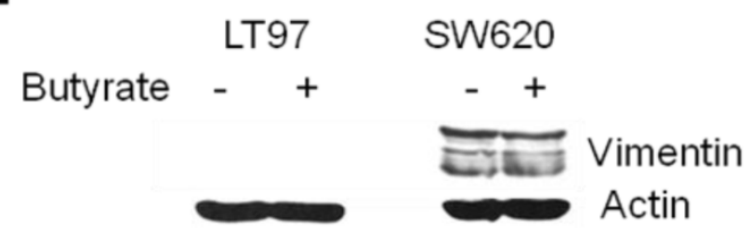

Figure 5. Western blot analysis of selected genes in LT97 and SW620 cells. LT97 or SW620 cells were mock treated (-) or treated with 5 mM butyrate (+) for 17.5 $\mathrm{hr}$ and then total protein was isolated. Western blot analysis was performed with the appropriate antibodies as described in Materials and Methods; the proteins analyzed are CBP (A), p300 (B), KLF4 (C), E-cadherin (D), c-myc (E), and vimentin (F). Actin was used as a loading control. Representative data are shown. 
The microarray data further indicated that basal expression of KLF4 was lower in LT97 cells compared to SW620 cells; however, butyrate treatment increased KLF4 expression in LT97 cells to levels higher than that observed in mock- or butyrate-treated SW620 cells. On the other hand, butyrate treatment did not increase KLF4 expression in SW620 cells. At the protein level, KLF4 expression was higher in LT97 cells compared to SW620 cells, both in the presence or absence of butyrate. Contrary to the array data, levels of KLF4 protein were increased in SW620 cells after butyrate treatment. Exposure of LT97 cells to butyrate resulted in expression of KLF4 protein levels approximately equal to these in mock-treated cells (Fig.5C).

Basal Wnt activity was lower in LT97 cells compared to CRC cells such as SW620; therefore, we hypothesized that factors that downregulate Wnt activity would be found at higher levels in LT97 compared to SW620 cells. Our array data revealed that two Wnt activity inhibitors, TLE1 (34) and E-cadherin (reviewed in ref. 35) were expressed at higher levels in LT97 cells, and that levels of E-cadherin were sharply upregulated in SW620 but not LT97 cells after exposure to butyrate. At the protein level, we observed that TLE1 expression levels were approximately equivalent in LT97 and SW620 cells (data not shown). On the other hand, we consistently observed higher basal expression of E-cadherin protein in LT97 compared to SW620 cells. Western blot analysis also showed enhanced expression of E-cadherin in SW620 cells after exposure to butyrate, while E-cadherin protein levels in LT97 cells were unchanged after exposure to this agent (Fig. 5D).

C-myc is a Wnt signaling-targeted gene, the product of which in part mediates the pro-proliferative action of constitutively activated Wnt signaling. Butyrate influences c-myc expression at several levels, resulting in a net downregulation of c-myc levels (36). Our array data indicated that c-myc is expressed at higher levels in SW620 cells than in LT97 cells, and is downregulated by butyrate in LT97 cells. Western blot analyses confirmed a marked downregulation of c-myc expression in butyrate-treated LT97 cells (Fig.5E). However, we did not observe significantly higher levels of c-myc protein in mock-treated SW620 compared to LT97 cells.

Vimentin is another Wnt signaling-targeted gene, and its product influences changes in cell shape and motility during the epithelial to mesenchymal transition that occurs during metastasis $(37,38$ and refs. therein). Microarray data indicated that vimentin is highly overexpressed in SW620 cells compared to LT97 cells in the presence and absence of butyrate. Western blot data supported this finding: under our blotting conditions, we detected vimentin protein in SW620, but not in LT97, cells (Fig.5F).

\section{Discussion}

The preventive role of fiber/butyrate against CRC is most likely exerted during early stage disease (7). We have reported that the ability of butyrate to induce growth arrest and apoptosis in CRC cells is associated with the ability of the agent to hyperactivate Wnt/beta-catenin signaling (5). Thus, we evaluated the effects of butyrate on Wnt signaling in LT97 microadenoma cells, which model early stage colonic neoplasia. LT97 cells exhibit a marked upregulation of Wnt/beta-catenin activity and apoptosis by butyrate (Fig.1). The greater-fold induction of Wnt activity by butyrate observed in LT97 cells (Fig.1A) compared to CRC cell lines (5) is consistent with the high sensitivity of LT97 cells to the growth suppressive effects of butyrate (14). Similarly, we found that LT97 cells exhibit a marked upregulation of caspase $3 / 7$ activity after exposure to butyrate (Fig.1B), consistent with our previous observation that CRC cell apoptosis induced by butyrate correlates with the degree of Wnt hyperactivation (5). Thus, butyrate treatment of LT97 cells results in a higher degree of Wnt hyperactivation and apoptosis than that observed in CRC cells.

We have previously observed cell type-specific effects of ICG-001 and ICG-001/butyrate cotreatment on caspase $3 / 7$ activation in CRC cells $(26,27)$. Thus, of four CRC cell lines studied (HCT-116, SW620, HCT-R, and HCT-15) only butyrate-resistant HCT-R cells exhibited a significant upregulation of caspase $3 / 7$ activity after exposure to ICG-001 alone. In addition, we observed a strong interference between ICG-001 and butyrate in the ability of these agents to induce caspase3/7 activity upon cotreatment of HCT-R and SW620 cells. In contrast, LT97 cells exhibited both (a) upregulation of caspase $3 / 7$ activity when exposed to ICG-001 alone; and (b) no interference between ICG-001 and butyrate, as cotreatment with these agents resulted in the highest levels of caspase 3/7 activity (Fig.2B). If LT97 cells are representative of early stage colonic neoplasia, these findings suggest that ICG-001-like agents promote apoptosis, and would be effective in suppressing early stages of colonic tumorigenesis. Furthermore, the lack of interference between the effects of ICG-001 and butyrate on LT97 cell apoptosis suggests that ICG-001-like agents can be effective in the context of a high fiber diet or therapeutic application of HDACis against early stage colonic neoplasia. In contrast, for certain established CRCs, ICG-001-like agents would be most effective administered alone, and/or in the context of fasting or a low fiber diet $(26,27)$.

Cotreatment of LT97 cells with butyrate, which 
upregulates Wnt signaling, and ICG-001, which represses Wnt signaling, led to the highest levels of caspase $3 / 7$ activity and lowest levels of cell proliferation (Figs.2B,C). Why does treatment with agents that upregulate (butyrate) and downregulate (ICG-001) Wnt activity cooperate to enhance Wnt activity-dependent effects on apoptosis and proliferation? One explanation is that ICG-001 specifically represses CBP-mediated Wnt signaling without affecting p300-mediated Wnt signaling $(18-21,26,27)$. It has been reported that CBP-mediated Wnt signaling promotes colonic cell proliferation; whereas, p300-mediated Wnt signaling promotes differentiation (16-21). Thus, ICG-001 specifically represses the CBP-mediated Wnt activity that is required for growth; whereas, leaving unaffected the p300-mediated Wnt signaling that promotes differentiation and apoptosis. In addition, while ICG-001 represses the overall levels of butyrate-mediated Wnt activity in LT97 cells, butyrate still retains the ability to induce a fold-increase in Wnt signaling (Fig.2A). We have previously demonstrated that the fold-change in Wnt activity, rather than the absolute level of Wnt activity, correlates with the effects of butyrate on apoptosis and proliferation (5). Thus, butyrate/ICG-001 cotreatment of LT97 cells represses CBP-mediated signaling pathways responsible for cell proliferation, while maintaining fold-induction of p300-mediated Wnt signaling that leads to reduced proliferation and enhanced apoptosis.

The effects of ICG-001 and butyrate on cell physiology may be influenced by differential expression of factors that affect apoptosis and cell cycle arrest, such as survivin and p21. We have previously shown that SW620 cells exhibit a high basal expression of the anti-apoptotic factor survivin, and survivin expression is suppressed by treatment with ICG-001 in the presence and absence of butyrate (26). Since the cotreatment of LT97 cells with ICG-001/butyrate results in efficient induction of apoptosis (Fig.2B), we investigated survivin expression. Unlike what was observed in CRC cells, survivin expression in LT97 cells was unaffected by ICG-001 treatment; however, survivin levels were downregulated by butyrate (Fig.2D). These findings suggest that approaches to downregulate survivin expression in early stage colonic neoplasia may enhance the efficacy of ICG-001 treatment.

Both ICG-001 and butyrate repress cell growth of LT97, and the combination of the two agents resulted in the greatest suppression of proliferation (Fig.2C). Expression data for p21, which promotes cell cycle arrest, are consistent with these findings. Butyrate upregulates p21 expression in LT97 cells, and this enhanced expression is maintained in the presence of
ICG-001 (Fig.2D). This finding contrasts with the observation that, in metastatic SW620 cells, cotreatment with ICG-001 interferes with the ability of butyrate to upregulate p21 (26). Thus, high p21 expression in LT97 cells cotreated with butyrate/ICG-001 correlates with the efficient suppression of cell proliferation. These findings suggest that growth arrest is an important mechanism whereby treatment with ICG-001, combined with dietary or pharmacological HDACis, can target early stage colonic neoplasia.

Using microarray analysis, we have identified a number of genes differentially expressed in LT97 vs. SW620 cells, in the presence or absence of butyrate. Some of these genes are involved in Wnt signaling, cell proliferation and metastasis (Table 1, Figs.4-6, and unpublished data) and may therefore influence neoplastic progression. The high CBP expression in LT97 cells (Fig.5A) is consistent with the marked sensitivity of this cell line to the repressive effects of ICG-001 on Wnt activity, proliferation and apoptosis (Fig.2). In contrast, there is less difference in p300 levels between LT97 and SW620 cells (Fig.5B). KLF4 expression was higher in LT97 cells (Fig.5C), consistent with the role of this transcription factor in repressing Wnt activity (basal Wnt activity is low in LT97 cells, Fig.1) and in promoting differentiation (LT97 cells being less tumorigenic than SW620). The higher levels of E-cadherin observed in LT97 cells (Fig.5D) are consistent with the role of the protein in suppressing basal Wnt activity (35). The reported effects of butyrate on c-myc expression in colonic cells are complex. Butyrate, which enhances Wnt signaling, increases c-myc transcriptional initiation; however, butyrate also causes a block in c-myc transcriptional elongation, so that the net effect on c-myc expression is often negative (36 and refs. therein). This is consistent with what is observed in our western blot analysis (Fig.5E). The enhanced vimentin expression in SW620 vs. LT97 cells (Fig.5F) was expected, and it correlates with the metastatic origin of the SW620 cell line.

In summary, our findings contribute to a better understanding of neoplastic progression. Since levels of CBP are higher in LT97 cells compared to SW620 cells (Fig.5A), one possibility is that a more advanced neoplastic phenotype (e.g., the SW620 phenotype) is characterized by relatively higher p300-mediated Wnt/catenin activity. However, that possibility is argued against by the finding that a number of CRC cell lines do not express p300 while continuing to express CBP (27 and references therein), as well as the observation that p300-mediated Wnt/catenin signaling is associated with differentiation, while CBP-mediated Wnt/catenin signaling is associated with CRC cell proliferation (16-21). Our working hypothesis (Fig.6) is that neoplastic progression is driven 
by a greater activity of CBP-mediated Wnt/catenin signaling compared to its p300-mediated counterpart. In addition, neoplastic progression is likely characterized by greater basal Wnt activity but less sensitivity to butyrate, as well as significant differences in gene expression (e.g., E-cadherin, vimentin).
This hypothesis can be evaluated through further in vitro cell culture analyses, as well as by ex vivo and in vivo studies, to identify changes in CBP/p300 activity and relevant gene expression that influences colonic neoplastic progression. Findings from these studies can assist the development of novel preventive and therapeutic approaches against CRC.

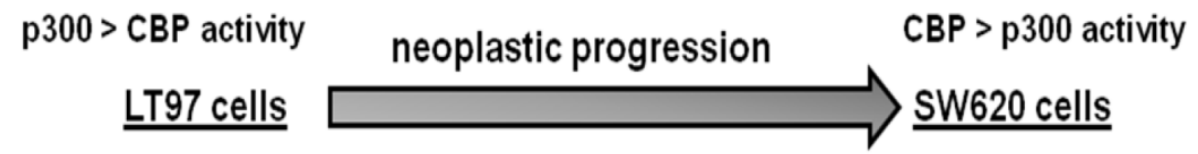

\section{E-cadherin \\ expression higher}

lowerbasalWnt activity, greater sensitivity to butyrate

\section{higherbasalWnt activity, less sensitivity to butyrate}

Fig. 6. Working hypothesis for neoplastic progression based on the LT97 and SW620 cell models. We hypothesize that early stage colonic neoplasia, modeled by LT97 cells, is characterized by: relatively higher p300 compared to CBP activity, lower basal Wnt activity but a greater sensitivity to the effects of butyrate on Wnt hyperactivation and the induction of apoptosis, and enhanced expression of gene products which suppress basal Wnt activity, such as E-cadherin. In contrast, advanced colonic neoplasia, modeled by metastatic SW620 cells, is characterized by relatively higher CBP compared to p300 activity, higher basal Wnt activity but less sensitivity to the effects of butyrate on Wnt hyperactivation and the induction of apoptosis, and enhanced expression of gene products which promote metastasis, such as vimentin. Alterations in cell signaling and gene expression that can transition more advanced neoplastic cells in the direction of a more LT97-type phenotype may have therapeutic value.

\section{Acknowledgements}

We thank Dr. Michael Kahn for supplying ICG-001. This work was supported by NIH NCI grant 1R15CA149589-01.

\section{Competing Interests}

The authors have declared that no competing interest exists.

\section{References}

1. Bingham SA, Day NE, Luben $R$, Ferrari $P$, Slimani $N$, Norat $T$, Clavel-Chapelon F, Kesse E, Nieters A, Boeing H, Tjonneland A, Overvad K, Martinzez C, Dorronsoro M, Gonzalez CA, Key TJ, Trichopolou A, Naska A, Vineis P, Tumino R, Krogh V, Bueno-de-Mesquita HB, Peeters PH, Berglund G, Hallmans G, Lund E, Skeie G, Kaaks R, Riboli E. Dietary fibre in food and protection against colorectal cancer in the European Prospective Investigation into Cancer and Nutrition (EPIC): an observational study. Lancet 2003; 361:1496-501.

2. Peters U, Sinha R, Chaterjee N, Subar AF, Ziegler RG, Kuldorff M, Bresalier R, Weissfeld JL, Flood A, Schtzkin A, Hayes RB. Dietary fibre and colorectal adenoma in a colorectal cancer early detection programme. Lancet 2003; 361:1491-5.

3. Bingham S. Mechanisms and experimental evidence relating dietary fibre and starch to protection against large bowel cancer. Proc Nutr Soc 1990; 49:153-71.

4. Bordonaro M, Mariadason JM, Aslam F, Heerdt BG, Augenlicht LH. Butyrate-induced apoptotic cascade in colonic carcinoma cells: modulation of the beta-catenin-Tcf pathway and concordance with effects of sulindac and trichostatin A but not curcumin. Cell Growth Differ 1999; 10:713-20.

5. Lazarova DL, Bordonaro M, Carbone R, Sartorelli AC. Linear relationship between WNT activity levels and apoptosis in colorectal carcinoma cells exposed to butyrate. Internat J Cancer 2004; 110:523-31.

6. Bordonaro M, Lazarova DL, Sartorelli AC. The activation of beta-catenin by WNT signaling mediates the effects of histone deacetylase inhibitors. Exp Cell Res 2007; 313:1652-66.

7. Bordonaro M, Lazarova D, Sartorelli AC. Hyperinduction of WNT signaling: a new paradigm for the treatment of colorectal cancer? Oncol Res 2008; 17:1-9.

8. Korinek V, Barker N, Morin PJ, Van Wichen D, De Weger R, Kinzler KW, Vogelstein B, Clevers H. Constitutive transcriptional activation by a beta-catenin-Tcf complex in APC-/- colon carcinoma. Science 1997; 275:1784-7.

9. Miyaki M, Iijima T, Kimura J, Yasuno M, Mori T, Hayashi Y, Koike M, Shitara $\mathrm{N}$, Iwama T, Kuroki T. Frequent mutation of $\beta$-catenin and APC genes in primary colorectal tumors from patients with hereditary nonpolyposis colorectal cancer. Cancer Res 1999; 59: 4506-9.

10. Li VSW, Ng SS, Boersema PJ, Low TY, Karthaus WR, Gerlach JP, Mohammed S, Heck AJR., Maurice MM, Mahmoudi T, Clevers H. Wnt signaling through inhibition of $\beta$-catenin degradation in an intact Axin1 complex. Cell 2012;149:1245-56.

11. Morin J, Sparks AB, Korinek V, Barker N, Clevers H, Vogelstein B, Kinzler $\mathrm{KW}$. Activation of beta-catenin-Tcf signaling in colon cancer by mutations in beta-catenin or APC. Science 1997; 275:1787-90.

12. Albuquerque $C$, Breukel $C$, van der Luijt R, Fidalgo P, Lage P, Slors FGM, Leitao CN, Fodde R, Smits R. The just-right signaling model: APC somatic mutations are selected based on a special level of activation of the beta-catenin signaling cascade. Hum Mol Genet 2002; 11:1549-60.

13. Richter M, Jurek D, Wrba F, Kaserer K, Wurzer G, Karner-Hanusch J, Marian B. Cells obtained from colorectal microadenomas mirror early premalignant growth patterns in vitro. Euro J Cancer 2002; 38: 1937-45.

14. Kautenberger T, Beyer-Sehlmeyer G, Festag G, Haag N, Kuhler S, Kuchler A, Weise A, Marian B, Peters WHM, Liehr T, Claussen U, Pool-Zobel BL. The gut fermentation product butyrate, a chemopreventive agent, suppresses glutathione S-transferase theta (hGSTT1) and cell growth more in human colon adenoma cells (LT97) than tumor (HT29) cells. J Cancer Res Clin Oncol 2005; 131:692-700.

15. Pool-Zobel BL, Salvarju V, Sauer J, Kautenberger T, Kiefer J, Richter KK, Soom M, Wolfl S. Butyrate may enhance toxicological defence in primary, adenoma, and tumor human colon cells by favorably modulating expression of glutathione S-transferases genes, an approach in nutrigenomics. Carcinogenesis 2005; 26: 1064-76.

16. Emami KH, Nguyen C, Ma H, Kim DH, Jeong KW, Eguchi M, Moon RT, Teo JL, Oh SW, Kim HY, Moon SH, Ha JR, Kahn M. A small molecule inhibitor of $\beta$-catenin/CREB-binding protein transcription. Proc Natl Acad Sci USA 2004; 101:12682-7.

17. McMillan M, Kahn M. Investigating WNT signaling: a chemogenomic safari. Drug Disc Today 2005; 10:1467-74.

18 Teo JL, Ma H, Nguyen C, lam C, Kahn M. Specific inhibition of CBP/ $\beta$-catenin interaction rescues defects in neuronal differentiation caused by presenilin-1 mutayion. Proc Natl Acad Sci 2005; 102:12171-6.

19. Ma H, Nguyen C, Lee KS, Kahn M. Differential roles for the coactivators CBP and $\mathrm{p} 300$ on $\mathrm{TCF} / \beta$-catenin-mediated survivin gene expression. Oncogene 2005; 24:3619-31.

20. Miyabayashi T, Teo JL, Yamamoto M, McMillian M, Nguyen C, Kahn M. WNT/ $\beta$-catenin/CBP signaling maintains long-term murine stem cell pluripotency. Proc Natl Acad Sci USA 2007; 104:5668-73.

21. Teo JL, Kahn M. The WNT signaling pathway in cellular proliferation and differentiation: a tale of two coactivators. Adv Drug Delivery Rev 2010; 62:1149-55.

22. Waltzer L, Bienz M. Drosophila CBP represses the transcription factor TCF to antagonize Wingless signaling. Nature 1998; 395:521-5. 
23. Hecht A, Vleminckx K, Stemmler MP, van Roy F, Kremler R. The p300/CBP acetyltransferases function as transcriptional coactivators of beta-catenin in vertebrates. EMBO J 2000; 19:1839-50.

24. Li J, Sutter C, Parker DS, Blauwkamp T, Fang M, Cadigan KM. CBP/p300 are bimodal regulators of WNT signaling. EMBO J 2007; 26:2284-94.

25. Levy L, Wei Y, Labalette C, Wu Y, Renard CA, Buendia MA, Neuveut C. Acetylation of beta-catenin by p300 regulates beta-catenin-Tcf4 interaction. Mol Cell Biol 2004; 24:3404-14.

26. Lazarova DL, Chiaro C, Wong T, Drago E, Rainey A, O'Malley S, Bordonaro M. CBP activity mediates effects of the histone deacetylase inhibitor butyrate on Wnt/catenin activity and apoptosis in colon cancer cells. J. Cancer 2013; 4: $481-90$.

27. Lazarova DL, Wong T, Chiaro C, Drago E, Bordonaro M. p300 influences butyrate-mediated WNT hyperactivation in colorectal cancer cells. J Cancer 2013; 4:491-501.

28. Evans PM, Chen X, Zhang W, Liu C. KLF4 interacts with $\beta$-catenin/TCF4 and blocks p300/CBP recruitment by $\beta$-catenin. Mol Cell Biol 2010; 30:372-81

29. Sellak H, Wu S, Lincoln TM. KLF4 and SOX9 transcription factors antagonize $\beta$-catenin and inhibit TCF-activity in cancer cells. Biochim Biophys. Acta 2012; 1823: 1666-675.

30. Hoffmeyer K, Raggioli A, Rudloff S, Anton R, Hierholzer A, Del Valle I, Hein K., Vogt R, and Kemler R. Wnt/ $\beta$-catenin signaling regulates telomerase in stem cells and cancer cells. Science 2012; 336: 1549-1554

31. Bowers EM, Yan G, Mukherjee C, Orry A, Wang L, Holbert MA, Crump NT, Hazzalin CA, Liszczak G, Yuan H, Larocca C, Saldanha SA, Abagyan R, Sun Y, Meyers DJ, Marmorstein R, Mahadevan LC, Alani RM, Cole PA. Virtual ligand screening of the $\mathrm{p} 300 / \mathrm{CBP}$ histone acetyltransferase: identification of a selective small molecule inhibitor. Chem and Biol 2010; 17:471-82.

32. Chiaro C, Lazarova DL, Bordonaro M. Tcf3 and cell cycle factors contribute to butyrate resistance in colorectal cancer cells. Biochem Biophys Res Commun 2012; 428:121-6.

33. Bordonaro M, Tewari S, Atamna W, Lazarova DL. The notch ligand Delta-like 1 integrates inputs from TGFBeta/Activin and Wnt pathways. Exp Cell Res 2011; 317: 1368-81.

34. Levanon D, Goldstein RE, Bernstein Y, Tang H, Goldenberg D, Stifani S, Paroush Z, Griner Y. Transcriptional repression by AML1 and LEF-1 is mediated by the TLE/Groucho corepressors. Proc Natl Acad Sci USA 1998; 95: 11590-5.

35. Wheelock MJ, Johnson KR. Cadherin-mediated cellular signaling. Curr Opin Cell Biol 2003; 15: 509-14.

36. Wilson AJ, Velcich A, Arango D, Kurland AR, Shenoy SM, Pezo RC, Levsky JM, Singer RH, Augenlicht LH. Novel detection and differential utilization of a c-myc transcriptional block in colon cancer chemoprevention. Cancer Res 2002; 62: 6006-10.

37. Gilles C, Polette M, Mestdagt M, Nawrocki-Raby B, Ruggeri P, Birembaur P, Foidart JM. Transactivation of vimentin by beta-catenin in human breast cancer cells. Cancer Res 2003; 63: 2658-64.

38. Mendez MG, Kojima S-I, Goldman RD. Vimentin induces changes in cell shape, motility, and adhesion during the epithelial to mesenchymal transition. The FASEB J 2010; 24: 1838-51.

\section{Author biography}

Michael Bordonaro received his $\mathrm{PhD}$ degree from Fordham University and did a post-doctoral fellowship at the Montefiore Medical Center at the Albert Einstein Cancer Center. It was during this period that Dr. Bordonaro discovered that the Wnt signaling pathway can be pharmacologically modulated, and that histone deacetylase inhibitors (HDACis) upregulate Wnt signaling in colorectal cancer cells in a manner associated with enhanced rates of apoptosis of these cells. While at Montefiore, Dr. Bordonaro was awarded an American Institute for Cancer Research Post-Doctoral Award grant for his work on HDACis and Wnt signaling. Dr. Bordonaro subsequently worked at the Yale University School of Medicine, where he continued his studies on the effects of HDACis on Wnt signaling and colonic cell physiology. In addition, at Yale, Dr. Bordonaro started work on Wnt-targeted genetic therapeutics against colorectal cancer. Dr. Bordonaro is currently an Associate Professor at The Commonwealth Medical College, and is continuing his research on these and other topics. 